\title{
Disorder Induced Diffusive Transport In Ratchets
}

\author{
M.N. Popescu ${ }^{1}$, C.M. Arizmendi ${ }^{1,2}$, A. L. Salas- Brito ${ }^{1,3}$, and F. Family ${ }^{1}$ \\ ${ }^{1}$ Department of Physics, Emory University, Atlanta, GA 30322, USA \\ ${ }^{2}$ Depto. de Física, Facultad de Ingeniería, Universidad Nacional de Mar del Plata, \\ Av. J.B. Justo 4302, 7600 Mar del Plata, Argentina \\ ${ }^{3}$ Laboratorio de Sistemas Dinámicos, Departamento de Ciencias Básicas, \\ Universidad Autónoma Metropolitana-Azcapotzalco, Apartado Postal 21-726, \\ Coyoacán 04000 D. F., México
}

(October 28, 2018)

\begin{abstract}
The effects of quenched disorder on the overdamped motion of a driven particle on a periodic, asymmetric potential is studied. While for the unperturbed potential the transport is due to a regular drift, the quenched disorder induces a significant additional chaotic "diffusive" motion. The spatio-temporal evolution of the statistical ensemble is well described by a Gaussian distribution, implying a chaotic transport in the presence of quenched disorder.
\end{abstract}

87.15.Aa, 87.15.Vv, 05.60.Cd, 05.45.Ac

Typeset using REVTEX 
Stochastic models known as thermal ratchets or correlation ratchets [1], in which a nonzero net drift velocity may be obtained from time correlated fluctuations interacting with broken symmetry structures [2], have recently received much attention. This interest is due to the wide range of possible applications of these models for understanding such systems as molecular motors [3], nano-scale friction [4], surface smoothening [5], coupled Josephson junctions [6], optical ratchets and directed motion of laser cooled atoms [7], mass separation and trapping schemes at the microscale [8]. Recently, spatial disorder in thermal ratchets has been shown to reduce the characteristic drift speed [9,10]. Little is known, however, about the effects of quenched spatial disorder on the regular or diffusive motion in otherwise periodic potentials 99 13].

Diffusion-like motion is observed in many types of deterministic systems. In particular, it has been shown that in deterministic chaotic systems, diffusion can be normal [14], with the mean-square displacement $\left\langle x^{2}\right\rangle$ proportional to time $t\left(\left\langle x^{2}\right\rangle \sim t\right)$, or it can be anomalous [15], with $\left\langle x^{2}\right\rangle \sim t^{\gamma}$, (enhanced for $\gamma>2$, dispersive for $1<\gamma<2$ ), or have a logarithmic time dependence $(\gamma=0)$ [16].

In the present work we report on an unusual behavior that occurs in the case of an overdamped ratchet subject to an external oscillatory drive: quenched disorder induces a normal diffusive transport in addition to the drift due to the external drive. For the parameter range considered, this process is observed even for very small perturbations. Moreover, this diffusive motion is enhanced by higher values of the quenched disorder. In fact, for high enough disorder the diffusive motion is of the same order of magnitude as the regular drift. The possibility of having large fluctuations, of the same order of magnitude as the average velocity, can be of great importance for a correct interpretation of experimental results. This may be of particular importance in studies of friction, in understanding the motion of nanoclusters or monolayers sliding on surfaces, as well as for designing particles separation techniques.

We consider the one-dimensional, overdamped motion of a particle (in dimensionless units) on a disordered ratchet: 


$$
\gamma \frac{d x}{d t}=\cos (x)+\mu \cos (2 x)+\Gamma \sin (\omega t)+\alpha \xi(x) .
$$

Here, $\gamma$ is the damping coefficient, $\Gamma$ and $\omega$ are, respectively, the amplitude and frequency of an external oscillatory forcing, and $\alpha \xi(x)$ is the force due to the quenched disorder. For the present study, $\xi(x) \in[-1,1]$ are independent, uniformly distributed random variables with no spatial correlations, and $\alpha \geq 0$ is the amount of quenched disorder. This corresponds to a piecewise constant force on the interval $[2 k \pi, 2(k+1) \pi), k \in \mathbb{Z}$. The unperturbed ratchet potential,

$$
U(x)=-\sin (x)-\mu \sin (2 x)
$$

has been the subject of extensive recent studies [17 19]. The quenched disorder term $(\alpha \neq 0)$ is expected to give either a more realistic representation of a real substrate or potential landscape, or to model fluctuations in DC current amplitude, as for arrays of Josephson junctions.

It is well known 17, 18 that in the absence of quenched disorder $(\alpha=0)$ there are unbounded solutions of Eq. (酉), provided that the driving amplitude $\Gamma$ is large enough. These solutions tend asymptotically to a constant average velocity independent of the initial conditions [18]. We have identified a set of parameters where, in the absence of disorder, the system shows non-zero current (regular transport). Specifically, we have selected $\gamma=1.0$, $\mu=0.25, \omega=0.1$, and we have studied the behavior for several values of $\Gamma$ with $\Gamma \geq 1.4$ (see below).

For $\alpha>0$ the periodicity of the unperturbed potential is destroyed as a result of the spatial randomness, and solutions of Eq. (11) begin to show a very complex behavior, including chaotic motion. The chaotic behavior is characterized by the rate of divergence of trajectories starting from very close initial conditions, in other words by the leading positive Lyapunov exponent. For a given, fixed realization of the quenched disorder, and for several values of $\Gamma \geq 1.4$, we have computed Lyapunov exponents over trajectories starting from origin. We have found positive Lyapunov exponents $\Lambda$ ranging from 2.55 , for $\Gamma=1.4$, to 3.22 
for $\Gamma=1.76$, which shows a very strong chaotic behavior. As a consequence of this chaotic behavior, the motion of the particle in the perturbed potential should be characterized by ensemble averages performed not only over realizations of disorder, but also over the spatial distribution of the positions of the particle in a given realization of the quenched disorder.

Numerical solutions of Eq. (四) were obtained using a variable step Runge-Kutta-Fehlberg method [20]. Averages were performed over ensembles of 5000 trajectories starting from different initial conditions very close to the origin $x=0$. The ensemble described above was left to evolve for 1000 external drive periods $T$, and every 10 periods the positions $x(t)$ were stored for further analysis.

We have first analyzed the motion in a given realization of disorder. In Fig. 11 we show results for the second moment, $C_{2}(t)=\left\langle(x(t)-\langle x(t)\rangle)^{2}\right\rangle$, where $\langle\ldots\rangle$ means average over the ensemble, as a function of the time $t$, for two different, fixed realizations of quenched disorder, and for two values of the disorder parameter $\alpha, \alpha=0.05$ (panel (a)), respectively $\alpha=0.10$ (panel $(\mathrm{b})$ ). The most striking feature is the fact that the second moment which is zero in the absence of disorder (corresponding to a purely deterministic motion), becomes non-zero in the presence of the perturbation. The non-zero second moment confirms the chaotic behavior mentioned above, showing a disorder-induced sensitive dependence on the initial conditions. It can be seen also that the time-dependence of the second moment is very complicated, and it is dependent on both the realization of quenched disorder and the amplitude $\alpha$ of disorder.

In order to perform averages over the realizations of disorder, we have used for each trajectory a different random sequence $\xi(x)$. In this way, the averages over the ensemble of trajectories include also averages over realizations of disorder. Figure 2 shows results for the first two moments, $C_{1}(t)=\langle x(t)\rangle$ and $C_{2}(t)=\left\langle(x(t)-\langle x(t)\rangle)^{2}\right\rangle$, as a function of the time $t$ for $\Gamma=1.5$ for several values of disorder parameter $\alpha$. In contrast to averages over a given "landscape", in this case both first and second moment show an asymptotic linear dependence on time $t, C_{1}(t) \simeq v(\alpha) t, C_{2}(t) \simeq D(\alpha) t$. We have considered several other values $1.40 \leq \Gamma \leq 1.76$, and we have observed the same linear behavior for all $\alpha$ values 
below a threshold value which depends on $\Gamma$. The quenched disorder induces fluctuations in the spatial position around the average value in our system, and the dynamics is no longer regular, but rather consists in a superposition of regular drift and diffusion-like chaotic motion. Moreover, even for reasonably small amounts of disorder, for example $\alpha=0.1$, it can be seen that these spatial fluctuations are of the same order of magnitude as the first moment, so the knowledge of a particular $x(t)$ no longer gives relevant information about the position of the center of mass of the distribution, an observation that can be of importance in studies of friction, particularly the sliding motion of clusters on surfaces 四.

The fluctuations in the position are characterics of chaotic behavior in deterministic systems. The description of the initial ensemble is then given by a probability distribution function $p_{t}(x)$, whose first two moments are linear in time as we have shown above. We have also calculated the higher order cumulants $C_{n}(t)$, for $n \leq 6$, and we have found that they increase slower than $t^{n / 2}$. Therefore, $p_{t}(x)$ is asymptotically a Gaussian, and it is determined by the first two moments [18]. In Figure 3 we show the distributions $P(z)$, where $z=x-\langle x\rangle$, for $\Gamma=1.50$ and two values of disorder parameter $\alpha=0.05$ (panel (a)), and $\alpha=0.10$ (panel (b)), at several times $t$, and the scaled distributions $f(y)=P(z) \times \sqrt{(} t)$, where $y=z / \sqrt{t}$; one can see that the distribution is indeed well aproximated by a Gaussian. This asymptotic Gaussian behavior also supports the conclusion that the motion is chaotic, as it was shown by Jung et al [18]. The reason for this chaotic behavior is the existence of discontinuities in the velocity at $x_{k}=2 \pi k$, where $k$ is an integer, introduced by the quenched disorder perturbation. These random kicks keep into a transitory regime the trajectories that in the absence of disorder would have asymptotically converged to the asymptotic constant speed state mentioned above. This "mixing" of transitory regimes causes the chaotic behavior, and we emphasize again that it is an effect due solely to the perturbation induced by quenched spatial disorder.

For several values of the external drive amplitude $\Gamma$, we have computed from the slopes of the first two moments, the drift velocity $v(\alpha)$, and the diffusion coefficient $D(\alpha)$, as functions of the amount of disorder $\alpha$. The results shown in Fig. 1 indicate that below a 
( $\Gamma$ dependent) threshold value of $\alpha$ the drift is slightly decreasing with increased quenched disorder, while the diffusion coefficient is steadily increasing and tends to saturate at high amounts of disorder. The fact that disorder has little effect on the drift motion is explained by the fact that the drift is a consequence of the initial asymmetry in the potential, and this asymmetry is only weakly influenced by small perturbations. We note here that there is no decrease in the diffusion coefficient over the range of disorder considered in this study. This is in contrast to the decrease of the diffusion coefficient observed in other systems [9],12]. The "divergence" of $D(\alpha)$ above the threshold can be understood if we consider the fact that $v(\alpha)$ decreases to zero. For large enough $\alpha$, some of the trajectories in the ensemble become bounded, and their contribution to the second moment is proportional to the displacement of the center of mass, thus with $t^{2}$. The number of bounded trajectories increases with time, as shown by the steady decrease of the drift velocity toward zero. The contribution to the second moment (fluctuations) of the $t^{2}$ term thus increases in time, and becomes dominant at late time, leading to the above mentioned "divergence" of $D(\alpha)$.

There is a number of experimental situations where small perturbations of a ratchet potential are relevant, including such systems as surface electromigration [5], dielectrophoretic trapping, and particle separation techniques [8]. Our preliminary results for the case of a non-negligible inertial term in Eq. (1) are qualitatively similar to the ones for the overdamped case, showing disorder induced chaotic diffusion. In this case, however, both the "diffusion coefficient" $D$ and the drift velocity $v$ depend on the mass of the particle. Based on the similarities mentioned, our results may be relevant for experiments where the mass dependence of the drift velocity or diffusion coefficient is essential. The efficiency of a nanoscale surface smoothening by an ac field suggested by Derényi et al [5] could be actually significantly smaller than theoretically predicted because of the chaotic diffusion, induced by the inherent "disorder" of a real surface, superimposed on the net downhill current. On the other hand, the rough, imperfect surface of the electrodes in the dielectrophoretic separation technique suggested by Gorre-Talini et al [8] can actually lead to a better efficiency of the process by superimposing the chaotic diffusion and drift on top of the thermal, Brownian 
motion. Moreover, the ac-separation techniques using a two-dimensional sieve discussed by Derényi and Astumian [8] can be modified in a very natural way to take advantage of the inherent imperfection of the two-dimensional structure. This can be done by replacing the Brownian diffusion along the drift direction with an additional ac-field along that direction.

Also, in this way one can fine tune both the drift velocity and the diffusion coefficient along the separation direction by a convenient choice of the ac-field parameters. The temperature can then be used for an independent tuning of the electrophoretic mobility, thus for the transverse displacement.

In summary we have shown that the addition of small amounts of quenched disorder in the equation of motion of a continuous time system induces a strong diffusive motion. In addition, we have found that the presence of small amounts of disorder slightly decreases the regular current (drift motion), but significantly increases the transport by chaotic diffusion. We have shown also that in the presence of disorder the spatial distribution of positions, averaged over the realizations of disorder, is described by a time-dependent Gaussian distribution, which is a signature of chaotic motion. These unexpected results may help in the interpretation of experimental results in studies of friction, particularly at the nanoscale, as well as in understanding transport processes in molecular motors or designing particle separation techniques.

\section{Acknowledgments}

This work was supported by grants from the Office of Naval Research, and from the Universidad Nacional de Mar del Plata. A. L. Salas-Brito wants to thank M. Mina and C. Ch. Ujaya for their friendly support and acknowledges the partial support of CONACyT through grant 1343P-E9607. 


\section{REFERENCES}

[1] C.R. Doering, Il Nuovo Cimento 17, 685 (1995).

[2] A. Ajdari and J. Prost, C. R. Acad. Sci. Paris 315, 1635 (1992); M. O. Magnasco, Phys. Rev. Lett. 71, 1477 (1993).

[3] J. Maddox, Nature 365, 203 (1993); 368, 287 (1994); 369, 181 (1994); S. Leibler, ibid. 370, 412 (1994); R. D. Astumian and M. Bier, Phys. Rev. Lett. 72, 1766 (1994); C. Doering, B. Ermentrout, and G. Oster, Biophysical Journal 69, 2256 (1995); R. D. Astumian and I. Derényi, Eur. Biophys. J. 27, 474 (1998).

[4] J. Krim, D. H. Solina, and R. Chiarello, Phys. Rev. Lett. 66, 181 (1991); J. B. Sokoloff, J. Krim, and A. Widom, Phys. Rev. B 48, 9134 (1993); L. Daikhin and M. Urbakh, Phys. Rev. E 49, 1424 (1994); C. Daly and J. Krim, Phys. Rev. Lett. 76, 803 (1996); M. R. Sørensen, K. W. Jacobsen, and P. Stoltze, Phys. Rev. B 53, 2101 (1996).

[5] I. Derényi, Choongseop Lee, and Albert-László Barabási, Phys. Rev. Lett. 80, 851 (1998).

[6] I. Zapata, R. Bartussek, F. Sols, and P. Hänggi, Phys. Rev. Lett. 77, 2292 (1996).

[7] L.P. Faucheux, L.S. Bourdieu, P.D. Kaplan, and A.J. Libchaber, Phys. Rev. Lett. 74, 1504 (1995); C. Mennerat-Robilliard, D. Lucas, S. Guibal, J. Tabosa, C. Jurczak, J.-Y. Courtois, and G. Grynberg, Phys. Rev. Lett. 82, 851 (1999).

[8] A.Adjari, D. Mukamel, L. Peliti, and J. Prost, J. Phys. I France 4, 1551 (1994); L. Gorre-Talini, J.P. Spatz, and P. Silberzan, Chaos 8, 650 (1998); I. Derényi and R. Dean Astunian, Phys. Rev. E 58, 7781 (1998).

[9] F. Marchesoni, Phys. Rev. E 56, 2492 (1997).

[10] T. Harms and R. Lipowsky, Phys. Rev. Lett. 79, 2895 (1997).

[11] E. Hernández-García, M.A. Rodríguez, L. Pesquera, and M. San Miguel, Phys. Rev. B 
42, 10653 (1990).

[12] G. Radons, Phys. Rev. Lett. 77, 4748 (1996).

[13] F. Iglói, L. Turban, and H. Rieger, Phys. Rev. E 59, 1465 (1999).

[14] T. Geisel and J. Nierwertberg, Phys. Rev. Lett. 48, 7 (1982); J. A. Blackburn and N. Grønbech-Jensen, Phys. Rev. E 53, 3068 (1996).

[15] M. F. Shlesinger, G. Zaslavsky, and J. Klafter, Nature 363, 31 (1993); J. Klafter, M. F. Shlesinger, G. Zumofen, Physics Today 49, 33 (1996); E. Barkai and J. Klafter, ibid. 79, 2245 (1997).

[16] E. Marinari, G. Parisi, D. Ruelle and P. Windey, Phys. Rev. Lett. 50, 1223 (1983); J. Krug and H. T. Dobbs, Phys. Rev. Lett. 76, 4096 (1996).

[17] R. Bartussek, P. Hänggi, and J.G. Kissner, Europhys. Lett., 28, 459 (1994).

[18] P. Jung, J.G. Kissner, and P. Hänggi, Phys. Rev. Lett. 76, 3436 (1996).

[19] B. Lindner, L. Schimansky-Geier, P. Reimann, P. Hänggi, and M. Nagaoka, Phys. Rev. E 59, 1417 (1999).

[20] W. H. Press, S. A. Teukolsky, W. T. Vetterling, and B. P. Flannery, Numerical Receipes in FORTRAN, (Cambridge University Press, Cambridge, 1995), pp. 704 - 716 


\section{FIGURES}

FIG. 1. Second moment $C_{2}(t)$ as a function of time for two different, fixed realizations of quenched disorder (solid, respectively dashed lines). The parameters used in Eq. (价) are $\gamma=1$, $\mu=0.25, \Gamma=1.76, \omega=0.1$, and $\alpha=0.05$ (panel (a)), respectively $\alpha=0.10$ (panel (b)).

FIG. 2. (a) First moment $C_{1}(t)$ as a function of time for several values of the amount of quenched disorder parameter $\alpha$. From top to bottom, $\alpha=0,0.05,0.10,0.15,0.20$. (b) Second moment $C_{2}(t)$ as a function of time for several values of the amount of quenched disorder parameter $\alpha$. From bottom to top, $\alpha=0,0.05,0.10,0.15,0.20$. The parameters used in Eq. (1) are $\gamma=1$, $\mu=0.25, \Gamma=1.50$, and $\omega=0.1$.

FIG. 3. Time evolution of the unscaled spatial distributions $P(z)$, where $z=x-\langle x\rangle$; from top to bottom, time $t=20 T, 40 T, \ldots, 100 T$. The inset shows the scaled distributions $P(z) \times \sqrt{t}$ vs $z / \sqrt{t}$. The solid line in the insets shows the theoretical, asymptotic Gaussian form. The parameters used in Eq. (11) are $\gamma=1, \mu=0.25, \Gamma=1.50, \omega=0.1$, and $\alpha=0.05$ (panel (a)), respectively $\alpha=0.10($ panel (b)).

FIG. 4. (a) Diffusion coefficient $D(\alpha)$ as a function of the amount of quenched disorder for $\Gamma=1.40,1.50,1.55,1.65,1.76$. (b) Drift velocity $v(\alpha)$ as a function of the amount of quenched disorder for $\Gamma=1.40,1.50,1.55,1.65,1.76$. The parameters are $\gamma=1, \mu=0.25, \omega=0.1$. 


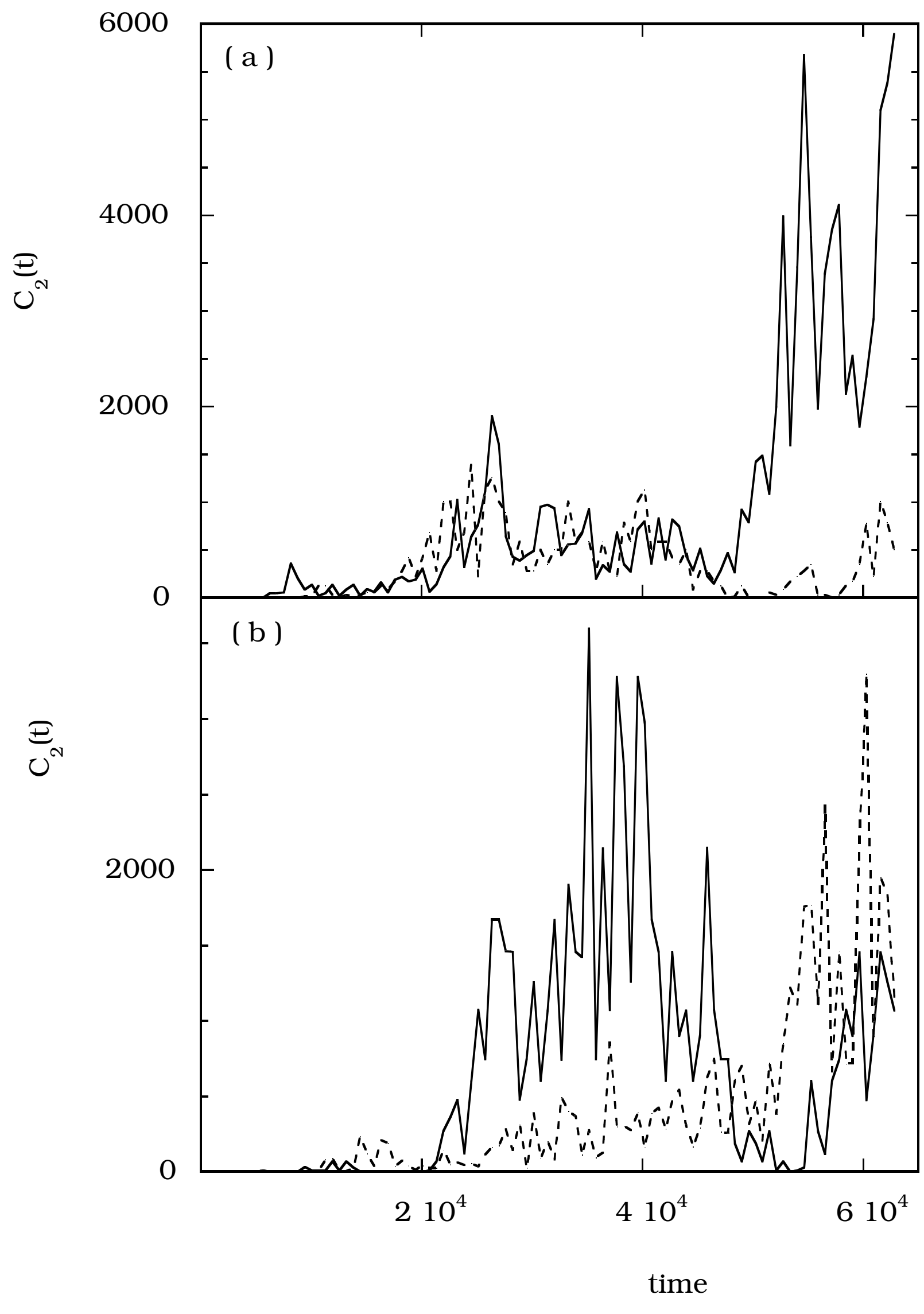

Figure 1 


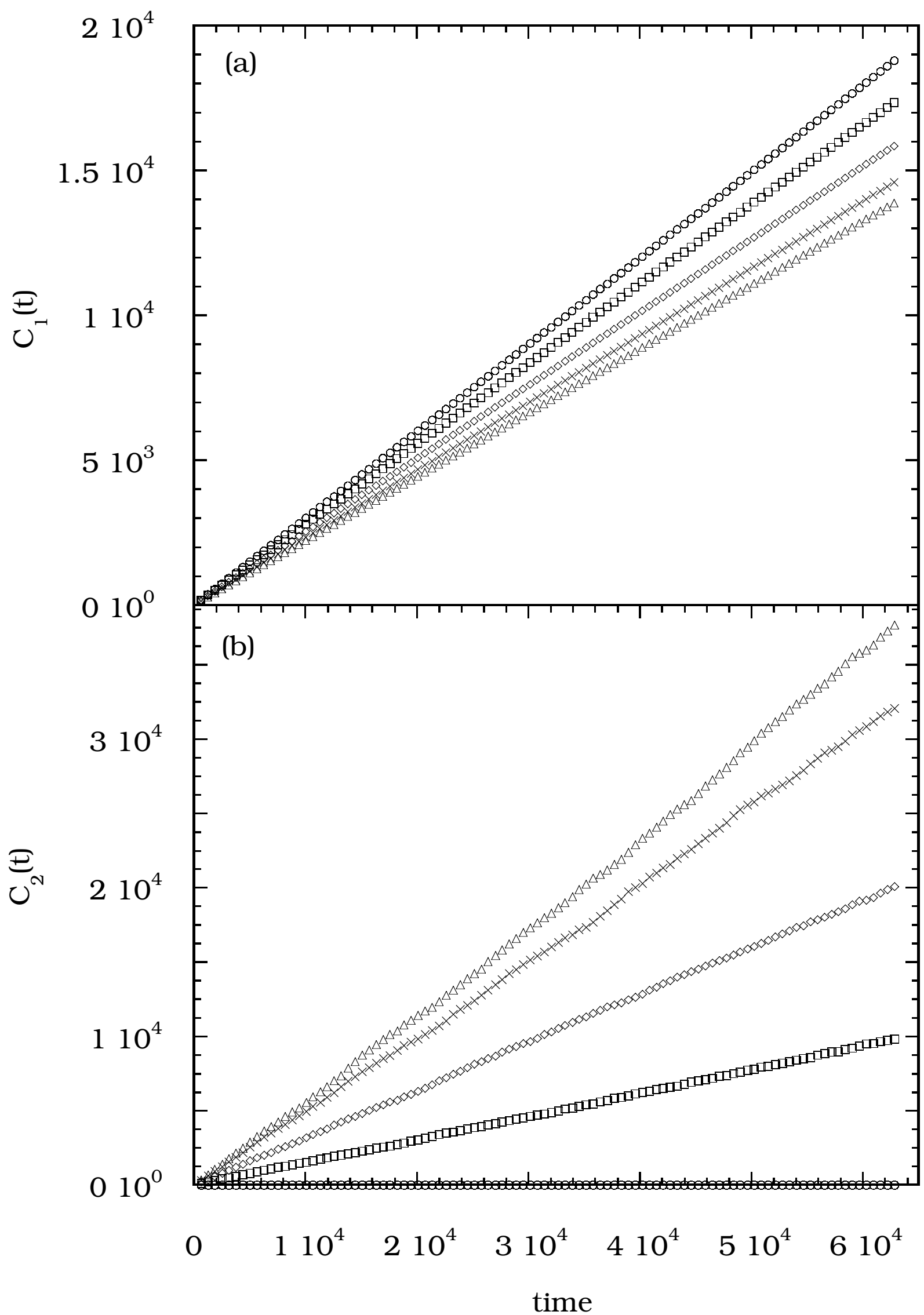

Figure 2 

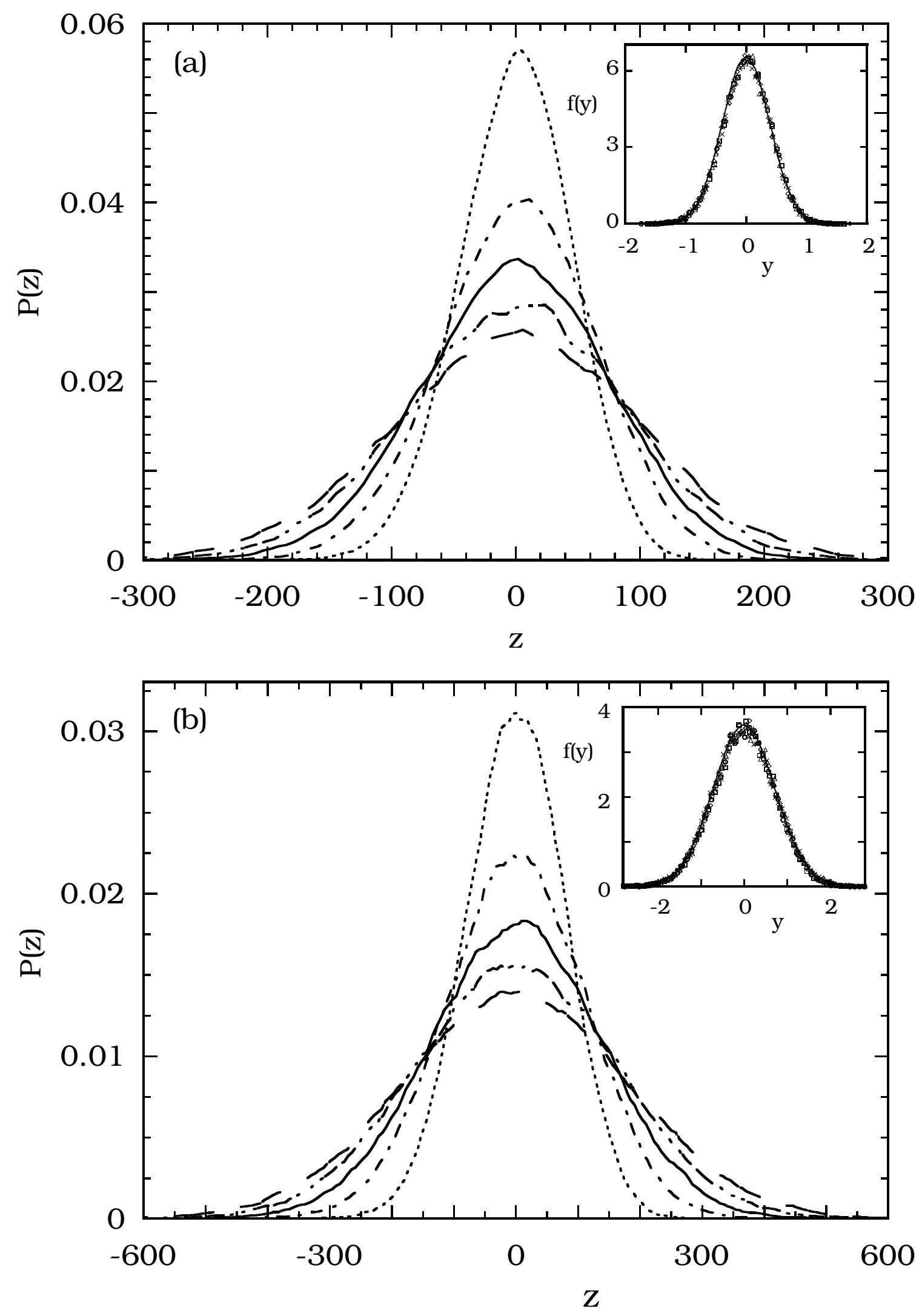

Figure 3 


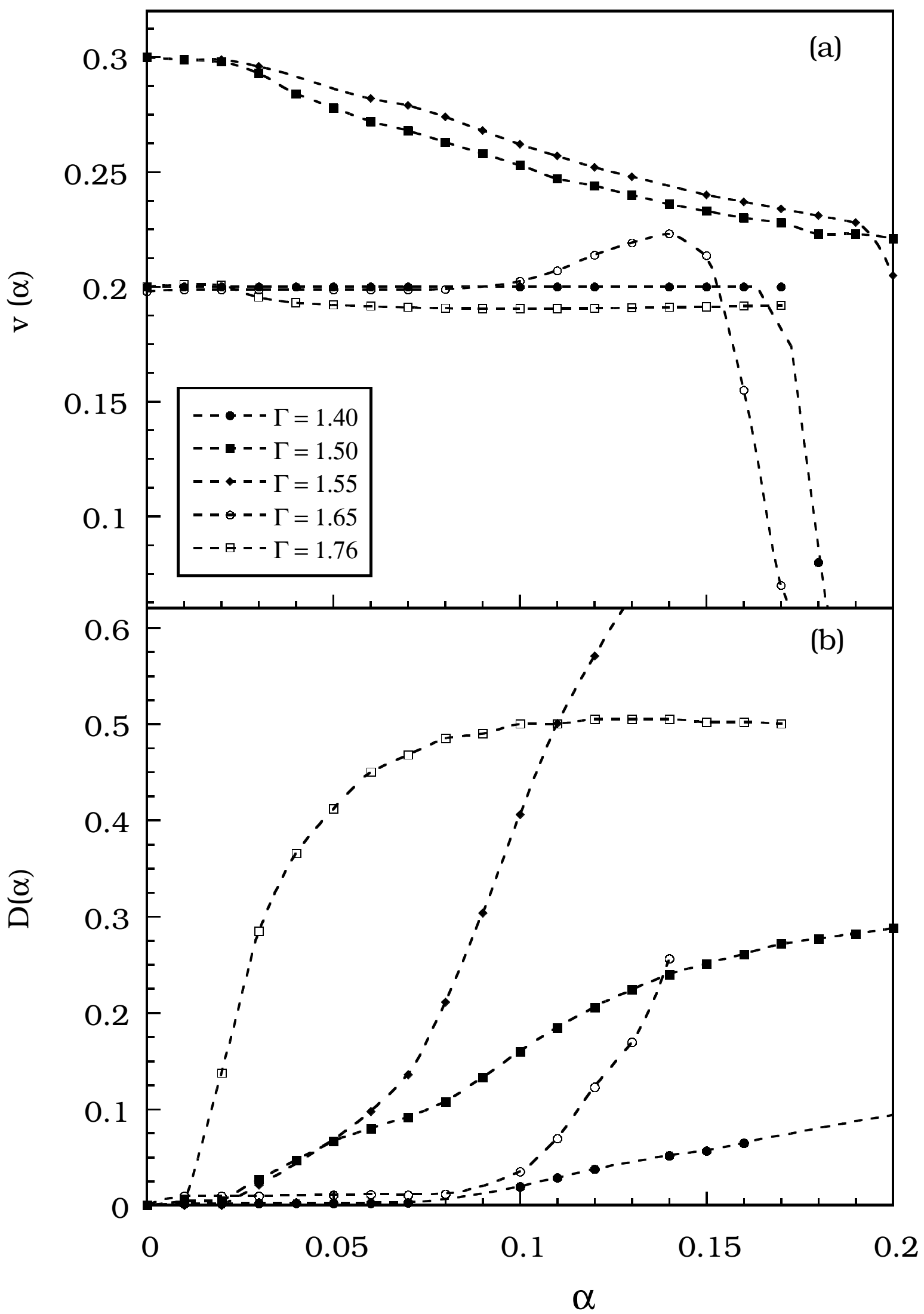

Figure 4 